\title{
OF PLANAR EULERIAN GRAPHS AND PERMUTATIONS
}

\author{
BY \\ GADI MORAN ${ }^{1}$
}

\begin{abstract}
Infinite planar Eulerian graphs are used to show that for $\nu>0$ the covering number of the infinite simple group $H_{\nu}=S / S^{\nu}$ is two.

Here $S$ denotes the group of all permutations of a set of cardinality $\aleph_{\nu}, S^{\nu}$ denotes its subgroup consisting of the permutations moving less than $\aleph_{\nu}$ elements, and the covering number of a (simple) group $G$ is the smallest positive integer $n$ satisfying $C^{n}=G$ for every nonunit conjugacy class $C$ in $G$.
\end{abstract}

1. Introduction. The covering number of a group $G$ is defined as the smallest positive integer $n$ such that $C^{n}=G$ holds for every nonunit conjugacy class $C$ in $G$. We write $\operatorname{cn}(G)=\omega$ when no such positive integer exists. See [AHSt, ACM] for further discussion and references

Obviously, if $\operatorname{cn}(G)<\omega$ then $G$ is simple and nonabelian. If $G$ is a finite nonabelian simple group, then $\operatorname{cn}(G)<\omega$ (see e.g. [AHSt]), but $\operatorname{cn}(G)$ rarely takes on the smallest conceivable value, namely two $[\mathbf{A C M}]$. On the other hand, the alternating group $A$ over any infinite set $B$ is an infinite (nonabelian) simple group satisfying $\operatorname{cn}(A)=\omega . A$ is also the first factor in the unique composition chain of the symmetric group $S=S_{B}$, consisting of all permutations of the set $B$. When $|B|=\aleph_{\nu}$, this chain is $1 \triangleleft A \triangleleft S^{0} \triangleleft S^{1} \triangleleft \cdots \triangleleft S^{\nu} \triangleleft S^{\nu+1}=S$, where $S^{\tau}$ denotes the group of all permutations of $B$ moving less than $\boldsymbol{\aleph}_{\tau}$ elements. Thus the composition factors are $A, \mathbf{Z}_{2}, H_{0}, \ldots, H_{\nu}$, where $\mathbf{Z}_{2}=\{0,1\}$ is the two-element group, and $H_{\tau}=S^{\tau+1} / S^{\tau}$. Rather surprisingly we have [ACM, Theorem 3.1].

THEOREM 1. Let $0<\nu$. Then $\operatorname{cn}\left(H_{\tau}\right)=2$ for $\tau=0, \ldots, \nu$.

A proof of this theorem for $0<\tau<\nu$ is presented in [ACM]. In this paper we shall establish it for the extreme cases, $\tau=0$ and $\tau=\nu$. The argument provided in [ACM] for the case $0<\tau<\nu$ combines Cayley's Representation Theorem with a theorem of G. A. Miller [Mi] (see §3). The proof we present here for the cases $\tau=0$, $\tau=\nu$ involves a direct combinatorial argument, where the construction of suitable infinite planar Eulerian graphs serves to establish the necessary inclusion relations

$$
C_{1} \subseteq C_{2} \cdot C_{3},
$$

where $C_{1}, C_{2}, C_{3}$ are conjugacy classes of the symmetric group over a countably infinite set. Relations of the form (1) form the core of any argument for Theorem 1.

Received by the editors March 15, 1983.

1980 Mathematics Subject Classification. Primary 20B30, 20E32, 05C25, 05C45; Secondary 20B22, 05C15, 05C10, 57M15.

${ }^{1}$ Supported in part by NSERC grant. 
The use of planar Eulerian graphs to establish such relations is both simple and extensive. In particular, it may replace the use of Miller's Theorem and Cayley's Representation Theorem, by providing a simple and purely elementary combinatorial argument for Theorem 1 . The method is developed in $\S 4$, where the central connection between planar Eulerian graphs and relations (1) is stated as Theorem 3.

Most of the paper is devoted to a detailed and largely self-contained argument for Theorem 1 with $\tau=\nu$, namely

THEOREM $1^{\prime}$. Let $\nu>0$. Then $\operatorname{cn}\left(H_{\nu}\right)=2$.

In $\S 2$ an outline of the argument is given. $\S 3$ reduces the argument to the proof of two propositions, 3.8 and 3.9 , which led us to the discovery of the method of planar Eulerian graphs treated in $\$ 4$. In $\$ 5$ this method is used to derive Propositions 3.8 and 3.9 from Proposition 5.1, which is established by making use of the existence of suitable planar Eulerian graphs.

$\S 6$ includes a proof of Theorem 1 for $\tau=0$. In fact, we show there that the extended covering number of $H_{0}$ is 3 if $\nu>0$ and 4 if $\nu=0$. This number is the smallest integer $k$ such that any product of $k$ nonunit classes in $H_{0}$ equals $H_{0}$. These results rely on a recent paper of Droste [D], as well as on $§ 5$. Roughly speaking, Droste's work is useful whenever at least two of the classes in (1) have at least one infinite orbit, while Proposition 5.1 is used in the other case. Theorem 1 for $\tau=0$ follows.

2. Outline of the argument. In this section we show how Theorem $1^{\prime}$ follows from a list of identities (1), where $C_{1}, C_{2}, C_{3}$ are conjugacy classes in $S_{0}$, the symmetric group over a countably infinite set.

We first develop some notation. $N=\{1,2, \ldots\}$ denotes the set of positive integers and $N^{+}=N \cup\left\{\boldsymbol{\kappa}_{0}\right\}$. A type is any cardinal-valued function $t$ defined on $N^{+} . n^{*}$ denotes the type defined by $n^{*}(n)=1, n^{*}(m)=0$ for $m \neq n$. For a type $t$ and a cardinal number $k$ a type $k \cdot t$ is defined by $(k \cdot t)(n)=k \cdot(t(n)), n \in N^{+}$. If $\left\{t_{i}\right.$ : $i \in I\}$ is an indexed family of types, then the sum $t=\sum_{i \in I} t_{i}$ is defined by the condition $t(n)=\sum_{i \in I} t_{i}(n), n \in N^{+}$. Thus, for every type $t$ we have $t=\sum_{n \in N^{+}} t(n)$. $n^{*}$. The class $T$ of all types forms a commutative semimodule over the class-semiring of cardinal numbers ${ }^{2}$ with the semigroup operation $t_{1}+t_{2}=\sum_{i \in\{1,2\}} t_{i}$.

For any $t \in T$, let $|t|$ denote the cardinal number defined by

$$
|t|=\sum_{n \in N^{+}} n \cdot t(n) .
$$

For any ordinal $\tau$, let $T_{\tau}$ denote the set of all types $T$ with $|t|<\boldsymbol{\aleph}_{\tau}$. Then $T_{\tau}$ forms a subsemigroup of $T$. We let $\equiv_{\tau}$ denote the congruence relation in $T$ modulo $T_{\tau}$, that is,

$$
t \equiv_{\tau} s \quad \text { iff } t=t_{0}+r, s=s_{0}+r \text { for some } r \in T, t_{0}, s_{0} \in T_{\tau} .
$$

Let $B$ be any set. Thus $|B|$ denotes its cardinality, $S_{B}$ denotes the group of all permutations of $B$, and $S_{B}^{\tau}$ its subgroup consisting of all permutations moving less

2 If the axiom of choice is not assumed, read "Alephs" instead of "cardinal numbers". 
than $\aleph_{\tau}$ elements. The subscript $B$ is omitted whenever the context allows. Permutations act on symbols from the left, and so $\eta \zeta$ means "first $\zeta$, then $\eta$ ".

For $b \in B, \xi \in S_{B}$, let $(b)_{\xi}=\left\{\xi^{m}(b): m=0, \pm 1, \pm 2, \ldots\right\}$ denote the $\xi$-orbit containing $b$. Thus $\left|(b)_{\xi}\right| \in N^{+}$for every $b \in B, \xi \in S_{B}$. With every $\xi \in S_{B}$ associate a type $\bar{\xi} \in T$ by letting $\bar{\xi}(n)$ be the cardinality of the set of $\xi$-orbits of cardinality $n$, that is,

$$
\bar{\xi}(n)=\left|\left\{(b)_{\xi}: b \in B,\left|(b)_{\xi}\right|=n\right\}\right| .
$$

Notice that $|\bar{\xi}|=|B|$ holds for every $\xi \in S_{B}$. Conversely, whenever $t \in T$ and a set $B$ satisfies $|B|=|t|$, there is a $t$-permutation $\xi$ in $S_{B}$, i.e., a $\xi \in S_{B}$ satisfying $\bar{\xi}=t$.

Let $\left\{B_{i}: i \in I\right\}$ be a partition of $B$, and let $\varphi_{i} \in S_{B_{i}}$ for $i \in I$. Then $\varphi=\sum_{i \in I} \varphi_{i}$ denotes the permutation of $B$ satisfying $\varphi \mid B_{i}=\varphi_{i}$ for all $i$ (where $\varphi \mid B_{i}$ denotes the restriction of $\varphi$ to the set $B_{i}$ ). Obviously, $\bar{\varphi}=\sum_{i \in I} \bar{\varphi}_{i}$. If, in addition, $\psi_{i} \in S_{B_{i}}$ for all $i \in I$ and $\psi=\sum_{i \in I} \psi_{i}$, then $\varphi \psi=\sum_{i \in I} \varphi_{i} \psi_{i}$. If $I=\{1, \ldots, n\}$, then we write $\varphi_{1}+$ $\cdots+\varphi_{n}$ for $\sum_{i \in I} \varphi_{i}$.

Let $\sim{ }_{G}$ denote the conjugacy relation in the group $G$, and let $[g]_{G}$ denote the conjugacy class of $g \in G$ in $G$; again, the subscript $G$ is omitted whenever the context allows.

The following propositions are well known.

Proposition 2.0 [S, 1.3.11]. Let $B$ be any set, $\xi, \eta \in S_{B}$. Then $\xi \sim \eta$ iff $\bar{\xi}=\bar{\eta}$.

Proposition 2.1 [M, Theorem 4]. Let $|B|=\aleph_{\nu}, 0<\tau \leqslant \nu, \xi, \eta \in S_{B}$. Then $\xi S^{\tau} \sim \eta S^{\tau}$ iff $\bar{\xi} \equiv{ }_{\tau} \bar{\eta}$.

Let $t$ be a type, $|t|=\aleph_{\nu}$, and let $0 \leqslant \tau \leqslant \nu$. We say that $t$ is $\tau$-proper iff:

(1) $t(1)=0$ or $t(1)=\boldsymbol{\aleph}_{\nu}$,

(2) $t(n)=0$ or $t(n) \geqslant \boldsymbol{\aleph}_{\tau}, n>1$.

Let $|B|=\aleph_{\nu}, \xi \in S_{B}$. We say that $\xi$ is $\tau$-proper iff $\bar{\xi}$ is $\tau$-proper. Notice that $\tau$-properness implies $\tau^{\prime}$-properness for $\tau^{\prime}<\tau$.

Proposition 2.1 implies

Proposition 2.2. Let $|B|=\aleph_{\nu}, 0 \leqslant \tau<\nu, \eta \in S_{B}$. Then there is a $\tau$-proper $\xi \in S_{B}$ with $\xi S^{\nu} \sim \eta S^{\nu}$.

Proof. Let $\xi \in S_{B}$ satisfy $\bar{\xi}(n)=0$ if $n=1$ and $\bar{\eta}(1)<\aleph_{\nu}$, or $n>1$ and $\bar{\eta}(n)<\boldsymbol{\aleph}_{\tau}$. Let $\bar{\xi}(n)=\bar{\eta}(n)$ otherwise. Then $\xi$ is $\tau$-proper, $\bar{\xi} \equiv_{\nu} \bar{\eta}$ and so $\xi S^{\nu} \sim \eta S^{\nu}$.

REMARK. If $\aleph_{\nu}$ is not the sum of countably many smaller cardinals, then in Proposition $2.2, \xi$ may be chosen to satisfy $\bar{\xi}(n) \in\left\{0, \aleph_{\nu}\right\}$ for all $n \in N^{+}$.

We are now in position to reduce Theorem $1^{\prime}$ to a theorem about the square of 0-proper classes of $S$. Indeed, if $|B|=\aleph_{\nu}$ then for all $\xi, \eta, \zeta \in S_{B},\left[\xi S^{\nu}\right]_{H_{\nu}} \subseteq\left[\eta S^{\nu}\right]_{H_{\nu}}$ $\cdot\left[\zeta S^{\nu}\right]_{H_{\nu}}$ follows from $[\xi] \subseteq[\eta] \cdot[\zeta]$. Thus, Theorem $1^{\prime}$ follows from

TheOREM 2. Let $|B|=\aleph_{\nu}, \nu>0$, and let $\xi, \eta \in S_{B}$ be 0 -proper permutations, where $\eta$ moves $\aleph_{\nu}$ elements. Then $\xi \in[\eta]^{2}$. 
Before presenting an argument for Theorem 2, it will be convenient to develop our notation, so that the relation (1) is modelled also in the realm of types.

If $C_{1}, C_{2}, C_{3}$ are conjugacy classes of a group $G$, let us write $P\left(C_{1}, C_{2}, C_{3}\right)$ if $C_{1} \subseteq C_{2} \cdot C_{3}$. Since $C_{2} \cdot C_{3}=C_{3} \cdot C_{2}$, we have $P\left(C_{1}, C_{2}, C_{3}\right)$ iff $P\left(C_{1}, C_{3}, C_{2}\right)$. Since $C_{1} \subseteq C_{2} \cdot C_{3}$ iff $C_{2} \subseteq C_{1} \cdot C_{3}^{-1}$, we have $P\left(C_{1}, C_{2}, C_{3}\right)$ iff $P\left(C_{2}, C_{1}, C_{3}^{-1}\right)$. It follows that if $C=C^{-1}$ holds for every class in $G$ then $P$ is a symmetric three-place relation on the set of $G$-classes. Since $C=C^{-1}$ holds for every class of $S$ (see Proposition 2.0), $C=C^{-1}$ holds also for any class in any homomorphic image of $S$, and so $P$ is a symmetric relation on the classes of $S$ as well as on the classes of $H_{\nu}$ (and in fact, on those of $H_{\tau}, 0 \leqslant \tau \leqslant \nu$ ).

We now extend $P$ to denote a three-place relation on any group $G$, setting $P\left(g_{1}, g_{2}, g_{3}\right)$ iff $P\left(\left[g_{1}\right],\left[g_{2}\right],\left[g_{3}\right]\right)$; and we extend $P$ to denote a symmetric relation on types in the natural way; namely, $P(r, s, t)$ holds for $r, s, t \in T$ iff $P(\xi, \eta, \zeta)$ holds for some permutations $\xi, \eta, \zeta$ with $\bar{\xi}=r, \bar{\eta}=s$ and $\bar{\zeta}=t$. Thus $P(r, s, t)$ holds iff $\xi=\eta \zeta$ holds for some $r$-permutation $\xi$, s-permutation $\eta$ and $t$-permutation $\zeta$.

Theorem 2 is restated now as

THEOREM $2^{\prime}$. Let $\nu>0$. Let $r, s \in T$ be 0-proper types, $|r|=|s|=\sum_{n>1} n \cdot s(n)=$ $\aleph_{\nu}$. Then $P(r, s, s)$ holds.

Theorem $2^{\prime}$ is a consequence of the following lemmas, the first of which summarizes the most useful properties of $P$ as a three-place relation on types.

LEMMA 1 (SyMmeTry, HOMOGENEITY AND SUPERADDITIVITY OF $P$ ). 1. $P\left(t_{1}, t_{2}, t_{3}\right)$ iff $P\left(t_{\theta(1)}, t_{\theta(2)}, t_{\theta(3)}\right)$ for any permutation $\theta$ of $\{1,2,3\}$.

2. $P(r, s, t)$ implies $p(k \cdot r, k \cdot s, k \cdot t)$ for any cardinal $k$.

3. $P\left(r_{i}, s_{i}, t_{i}\right)$ for all $i \in I$ implies $P(r, s, t)$, where $r=\sum_{i \in I} r_{i}, s=\sum_{i \in I} s_{i}$, $t=\sum_{i \in I} t_{i}$.

Proof. 1 was discussed above, and 2 follows from 3. To prove 3, let $B$ be any set of cardinality $|r|=\sum_{n \in N^{+}} n \cdot r(n)$, and let $\xi \in S_{B}$ satisfy $\bar{\xi}=r$. By $r=\sum_{i \in I} r_{i}$, there is a partition $\left\{B_{i}: i \in I\right\}$ of $B$ into $\xi$-invariant subsets such that $\xi_{i}=\xi \mid B_{i} \in S_{B_{i}}$ satisfies $\bar{\xi}_{i}=r_{i}$ for each $i \in I$. By $P\left(r_{i}, s_{i}, t_{i}\right),\left|r_{i}\right|=\left|s_{i}\right|=\left|t_{i}\right|=\left|B_{i}\right|$ holds, and there are $\eta_{i}, \zeta_{i} \in S_{B_{i}}$ with $\bar{\eta}_{i}=s_{i}, \bar{\zeta}_{i}=t_{i}$, satisfying $\xi_{i}=\eta_{i} \zeta_{i}$. Let $\eta=\sum_{i \in I} \eta_{i}, \zeta=\sum_{i \in I} \zeta_{i}$. Then $\eta, \zeta \in S_{B}, \bar{\eta}=\sum_{i \in I} s_{i}=s, \bar{\zeta}=\sum_{i \in I} t_{i}=t$, and $\eta \zeta=\sum_{i \in I} \eta_{i} \zeta_{i}=\sum_{i \in I} \xi_{i}=\xi$. Hence, $P(r, s, t)$ holds.

LEMMA 2. $P\left(k \cdot 1^{*}, s, s\right)$ holds for every cardinal number $k$ and type $s$ with $|s|=k$.

Proof. This is a restatement of the fact that every permutation is conjugate to its inverse.

The following two lemmas form the core of the proof of Theorem 1. Their proofs are discussed in $\S \S 3$ and 5. Lemma 3 appears also in [ACM].

Lemma 3. $P\left(\boldsymbol{\aleph}_{0} \cdot n^{*}, \boldsymbol{\aleph}_{0} \cdot m^{*}, \boldsymbol{\aleph}_{0} \cdot l^{*}\right)$ holds for all $n, l, m \in N^{+}, 1<n, l, m$. 
Lemma 4. $P\left(\boldsymbol{\aleph}_{0} \cdot n^{*}, \boldsymbol{\aleph}_{0} \cdot\left(1^{*}+m^{*}\right), \boldsymbol{\aleph}_{0} \cdot\left(1^{*}+m^{*}\right)\right)$ holds whenever $n, m \in N^{+}$, $m$ greater than 1 .

Proof of Theorem 2'. Let $r, s$ be types satisfying $|r|=|s|=\sum_{1<n} s(n)=\boldsymbol{\aleph}_{\nu}$, $r(n)=0$ or $r(n)$ infinite, $s(n)=0$ or $s(n)$ infinite for $n \in N^{+}$, and $s(1)=0$ or $s(1)=\boldsymbol{\aleph}_{\nu}$ (these assumptions hold in Theorem $2^{\prime}$ ). Let $I$ be a set of cardinality $\boldsymbol{\aleph}_{\nu}$. By our assumptions, we can define $n_{i}, m_{i}$ for $i \in I$ so that $n_{i}, m_{i} \in N^{+}, 1<m_{i}$, and $\left|\left\{i: n_{i}=n\right\}\right|=n r(n)=r(n),\left|\left\{i: m_{i}=m\right\}\right|=m s(m)=s(m)$ for $n, m \in N^{+}, m>1$.

Define $r_{i}, s_{i}$ for $i \in I$ by $r_{i}=\boldsymbol{\aleph}_{0} \cdot n_{i}^{*}$, and $s_{i}=\boldsymbol{\aleph}_{0} \cdot m_{i}^{*}$ if $s(1)=0, s_{i}=\boldsymbol{\aleph}_{0}$. $\left(1^{*}+m_{i}^{*}\right)$ if $s(1)=\aleph_{\nu}$. Then we have $r=\sum_{i \in I} r_{i}, s=\sum_{i \in I} s_{i}$. Also, by Lemmas 2-4 we have $P\left(r_{i}, s_{i}, s_{i}\right)$ for all $i \in I$. Hence, by Lemma $1, P(r, s, s)$.

3. Some preliminary observations. In this section we reduce the proof of Lemma 4 to that of Propositions 3.8 and 3.9 which are proved in $\$ 5$. The method of proof involves repeated use of the properties of $P$ listed in Lemma 1, in order to establish the $P$-relations in Lemmas 2 and 3 from simpler ones. Lemma 3 is proved in [ACM]. For the convenience of the reader we sketch its proof below.

Proposition 3.1 (G. A. Miller, 1900 [Mi]). Let n, m, l be positive integers greater than one. Then there exists a finite group $G$ with elements $g_{n}, g_{m}, g_{l}$ in $G$ of orders $n, m, l$, respectively, such that $g_{n}=g_{m} g_{l}$.

Proposition 3.1 was rediscovered several times since 1900. (The author rediscovered it in 1981, in the course of establishing Theorem 1.) See e.g. [F].

Assume now that $G$ mentioned in Proposition 3.1 satisfies $|G|=k$. Then $k=n^{\prime}$. $n=m^{\prime} \cdot m=l^{\prime} \cdot l ; n^{\prime}, l^{\prime}, m^{\prime} \in N$. Let $g_{n}^{\circ}, g_{m}^{\circ}, g_{l}^{\circ}$ be the permutations of $G$ obtained from $g_{n}, g_{m}, g_{l}$ in the proof of Cayley's Representation Theorem; that is, let $g_{n}^{\circ}(x)=g_{n} x, x \in G$. Then obviously $\bar{g}_{n}^{\circ}=n^{\prime} \cdot n^{*}$, and similarly $\bar{g}_{m}^{\circ}=m^{\prime} \cdot m^{*}$, $\bar{g}_{l}^{\circ}=l^{\prime} \cdot l^{*}$. Also $g_{n}^{\circ}=g_{m}^{\circ} g_{l}^{\circ}$. Thus we obtain from Proposition 3.1

Proposition 3.2. Let $n, m, l \in N, 1<n, l, m$. Then there are $n^{\prime}, l^{\prime}, m^{\prime} \in N$ such that $P\left(n^{\prime} \cdot n^{*}, m^{\prime} \cdot m^{*}, l^{\prime} \cdot l^{*}\right)$ holds.

It is not hard to extend Proposition 3.1 to the case where $n, m, l$ are allowed to assume also the value $\boldsymbol{\aleph}_{0}$ (see [ACM]). It follows that Proposition 3.2 still holds if we substitute $N^{+}$for $N$. Now Lemma 3 follows, since $P\left(\boldsymbol{\aleph}_{0} \cdot n^{*}, \boldsymbol{\aleph}_{0} \cdot m^{*}, \boldsymbol{\aleph}_{0} \cdot l^{*}\right)$ for $n, m, l \in N^{+}, 1<n, l, m$, follows from 3.2 by the homogeneity of $P$.

Let us turn now to Lemma 4 . It will be convenient to introduce the abbreviation:

$$
Q(n, m) \text { iff } P\left(\boldsymbol{\aleph}_{0} \cdot n^{*}, \boldsymbol{\aleph}_{0} \cdot\left(1^{*}+m^{*}\right), \boldsymbol{\aleph}_{0} \cdot\left(1^{*}+m^{*}\right)\right) .
$$

Thus, Lemma 4 is restated as

LEMMA 4'. $Q(n, m)$ holds whenever $n, m \in N^{+}, m>1$.

We establish first several special cases.

Proposition 3.3. $Q(1, m), 1 \leqslant m \leqslant \boldsymbol{\aleph}_{0}$.

This follows from Lemma 2. 
Proposition 3.4. $Q(2, m), m=2,3$.

Proof. (a) $Q(2,2)$ : indeed, with $B=N$ we have

$$
(1,2)(3,4)(5,6)(7,8) \cdots=[(1,2)(5,6) \cdots][(3,4)(7,8) \cdots] .
$$

(b) $Q(2,3)$ : indeed, with $B=\{1,2,3,4\}$ we have $(1,2)(3,4)=(2,4,3)(2,1,3)$ whence $Q(2,3)$ follows by Lemma 1 .

Proposition 3.5. ${ }^{3} Q(n, 2), 1 \leqslant n \leqslant \boldsymbol{\aleph}_{0}$.

Proof. The following are well known [S, 10.1.17]:

$$
\begin{aligned}
& P\left((2 m+1)^{*}, 1^{*}+m \cdot 2^{*}, 1^{*}+m \cdot 2^{*}\right), \quad 1 \leqslant m<\boldsymbol{\aleph}_{0}, \\
& P\left((2 m)^{*}, 2 \cdot 1^{*}+(m-1) \cdot 2^{*}, m \cdot 2^{*}\right), \quad 1 \leqslant m<\aleph_{0}, \\
& P\left(\boldsymbol{\aleph}_{0}^{*}, 1^{*}+\boldsymbol{\aleph}_{0} \cdot 2^{*}, \boldsymbol{\aleph}_{0} \cdot 2^{*}\right) .
\end{aligned}
$$

$Q(n, 2)$ for $1 \leqslant n \leqslant \aleph_{0}$ follows by Lemma 1 .

Proposition 3.6. ${ }^{3,4} Q\left(n, \aleph_{0}\right), 1<n \leqslant \aleph_{0}$.

Proof. Assume first $n<\aleph_{0}$. Let $Z=\{0,1,-1,2,-2, \ldots\}$ denote the set of integers, and let $B=Z \times\{0,1, \ldots, n-1\}$. Let $\dot{+}$ denote addition modulo $n$ on $\{0,1, \ldots, n-1\}$.

Define $\xi, \eta, \zeta \in S_{B}$ by setting

$$
\begin{array}{ll}
\xi(j, i)=(j, i+1), & j \in \mathbf{Z}, i \in\{0, \ldots, n-1\}, \\
\eta(j, 0)=(j-1,0), & j \in \mathbf{Z}, \\
\eta(j, i)=(j, i), & j \in \mathbf{Z}, i \in\{1, \ldots, n-1\}, \\
\zeta(j, i)=(j, i+1), & j \in \mathbf{Z}, i \in\{0, \ldots, n-2\}, \\
\zeta(j, n-1)=(j+1,0) & j \in \mathbf{Z} .
\end{array}
$$

Then $\xi=\eta \zeta, \quad \bar{\xi}=\aleph_{0} \cdot n^{*}, \bar{\eta}=\aleph_{0} \cdot 1^{*}+\boldsymbol{\aleph}_{0}^{*}$ and $\bar{\zeta}=\aleph_{0}^{*}$. Thus, we have $P\left(\boldsymbol{\aleph}_{0} \cdot n^{*}, \boldsymbol{\aleph}_{0} \cdot 1^{*}+\boldsymbol{\aleph}_{0}^{*}, \boldsymbol{\aleph}_{0}^{*}\right)$ and by Lemma 1 , we have $Q\left(n, \boldsymbol{\aleph}_{0}\right), 1<n<\boldsymbol{\aleph}_{0}$.

$Q\left(\boldsymbol{\aleph}_{0}, \boldsymbol{\aleph}_{0}\right)$ is a consequence of the obvious relation

$P\left(2 \cdot \boldsymbol{\aleph}_{0}^{*}, \boldsymbol{\aleph}_{0} \cdot 1^{*}+\boldsymbol{\aleph}_{0}^{*}, \boldsymbol{\aleph}_{0} \cdot 1^{*}+\boldsymbol{\aleph}_{0}^{*}\right)$.

Proposition 3.7. ${ }^{3} Q\left(\boldsymbol{\aleph}_{0}, m\right), 2<m<\boldsymbol{\aleph}_{0}$.

Proof. Let $B=\mathbf{Z} \times\{0,1, \ldots, m-1\}$. Let $\dot{+}$ denote addition modulo $m$ on $\{0, \ldots, m-1\}$. Define $\xi, \eta, \zeta \in S_{B}$ by setting for $j \in \mathbf{Z}, i \in\{0, \ldots, m-1\}$,

$$
\begin{aligned}
& \xi(j, i)=(j, i+1) \quad(i<m-1), \\
& \xi(j, m-1)=(j+1,0), \\
& \eta(2 j, i)=(2 j, i+1), \\
& \eta(2 j+1, i)=(2 j+1, i), \\
& \zeta(2 j+1, i)=(2 j+1, i+1), \\
& \zeta(2 j, i)=(2 j, i) .
\end{aligned}
$$

\footnotetext{
${ }^{3}$ See Figure 1 for a graphical proof.

${ }^{4}$ This proposition was obtained by A. B. Gray in 1960 [G]. We are grateful to the referee for calling our attention to this remark.
} 
Then

$$
\xi=\eta \zeta, \quad \bar{\xi}=\aleph_{0}^{*}, \quad \bar{\eta}=\bar{\zeta}=\boldsymbol{\aleph}_{0}\left(1^{*}+m^{*}\right),
$$

and so $P\left(\boldsymbol{\aleph}_{0}^{*}, \boldsymbol{\aleph}_{0} \cdot\left(1^{*}+m^{*}\right), \boldsymbol{\aleph}_{0} \cdot\left(1^{*}+m^{*}\right)\right)$ holds. $Q\left(\boldsymbol{\aleph}_{0}, m\right)$ follows by homogeneity of $P$.

In $\$ 5$ we shall prove

Proposition 3.8. $Q(n, m), 2<n, m<\boldsymbol{\aleph}_{0}$.

Proposition 3.9. $Q(2, m), 3<m<\boldsymbol{\aleph}_{0}$.

It follows from Propositions 3.3-3.9 that $Q(n, m)$ holds for all $1 \leqslant n \leqslant \boldsymbol{\aleph}_{0}$ and $1<m \leqslant \boldsymbol{\aleph}_{0}$. Thus Lemma $4^{\prime}$ is proved, and the proof of Theorem $1^{\prime}$ is complete.

4. Planar Eulerian graphs and permutations. Recall that for types $r, s, t, P(r, s, t)$ means that an $r$-permutation is a product of an $s$-permutation and a $t$-permutation (see $\$ 2$ for definitions). In this section a method to establish $P(r, s, t)$ by constructing suitable planar Eulerian graphs is developed. Briefly, the method is this:

Any planar Eulerian graph $G$ with a Black-White coloring of its plane regions defines three types $b_{G}, w_{G}, d_{G}$ by the edge length of boundaries of its black regions, of its white regions and the halves of its vertex degrees. We show that $P\left(b_{G}, d_{G}, w_{G}\right)$ holds. The details follow.

By a planar graph $G$ we shall mean, as usual, a pair $G=\left(V_{G}, E_{G}\right)$ where $V=V_{G}$ is a set of points in the plane-the set of vertices-and $E=E_{G}$ is the set of edges. Each edge $e$ is a subset of the plane satisfying $e=f([0,1])$, where $f$ is a continuous mapping of the closed unit interval $[0,1]$ into the plane such that $f \mid(0,1)$ is a homeomorphism. $f(0), f(1)$ are called the endpoints of $e$. The endpoints of $e \in E$ must belong to $V$ but need not be distinct (loops are allowed) and two distinct edges may have the same endpoints (multiplicity of edges (including loops) is allowed). Any edge meets $V$ by the set of its endpoints; and the intersection of two distinct edges is a set of vertices (so any two edges meet at most at common endpoints). In addition to those usual conditions, we require two conditions of local-finiteness.

(a) Every bounded set meets $V$ in a finite set.

(b) Every bounded set meets only finitely many edges.

The degree $d(v)$ of $v \in V$ is defined as the number of times $v$ occurs as an endpoint to an edge (so a loop contributes 2 to this count). It follows from (b) that every vertex $v$ has a finite degree.

We let $G_{*}=V \cup(\cup E)$ denote the set of points in $V$ or points in one of the edges (so $V \subseteq G_{*}$ and $e \subseteq G_{*}$ for every $e \in E$ ). We let $G_{*}^{c}$ denote the complement of $G_{*}$ in the plane. It follows from (a) and (b) that $G_{*}^{c}$ is an open set. We refer to its connected components as the regions of the graph $G$, or the $G$-regions.

By a planar Eulerian graph (PEG) we mean a planar graph $G$ satisfying the following two extra conditions.

(c) $G_{*}$ is connected.

(d) $d(v)$ is even for every $v \in V$. 
Let $G$ be a PEG and let $x \in e \in E_{G}$. Then any closed simple curve meeting $G^{*}$ only at $x$ encloses an open region $U$ disjoint from $G^{*}$. (Negate. Then by (a), (b), $U$ must witness a $G$-vertex of odd degree, contradicting (d).) Thus we have

Proposition 4.0 Let $G$ be a PEG. Then every edge e lies between two distinct G-regions.

[In other words, every region $U$ of a PEG is a regular open set: $U=$ int $c l U$.]

Let $C$ be a set of edges of a planar graph $G$. Then we say that $C$ is a circuit if $C$ has an enumeration without repetition $C=\left\{e_{i}: i \in A\right\}$, such that one of the following holds.

1. $A=\{0\}$ and $e_{0}$ is a loop.

2. Either (i) $A=\{0, \ldots, n-1\}$ for $n>1$, or (ii) $A=Z$; and for each $i \in A$, the set of endpoints of $e_{i}$ is $\left(e_{i-1} \cap e_{i}\right) \cup\left(e_{i} \cap e_{i+1}\right)$ (where $e_{-1}=e_{n-1}, e_{n}=e_{0}$ in case (i)).

For any subset $U$ of the plane, let $C(U)$ denote the set of edges included in the closure of $U$. If $G$ is an arbitrary plane graph and $U$ is a $G$-region, then little can be said of $C(U)$. If, however, $G$ is assumed to be Eulerian, $C(U)$ must be a circuit. Indeed, this is an immediate consequence of our definitions and Proposition 4.0. We state it as

Proposition 4.1. Let $G$ be a PEG, and let $U$ be a G-region. Then $C(U)$ is a circuit, and $\bigcup_{e \in C(U)}$ is the boundary of $U$.

We let $c(U)=|C(U)|$. Thus, $c(U)$ is the number of $G$-edges included in the closure of the $G$-region $U$ (in short, touching $U$ ). Obviously, $c(U) \in N^{+}$.

Let $G$ be a PEG. By a bicoloring of $G$ or $a B$-W-coloring of $G$ we mean a Black-White coloring of its regions; (that is, a mapping $f$ of the set of $G$-regions into the set of two colors $\{B=$ Black, $W=$ White $\}$ ) so that every edge $e$ lies between a black region and a white region. Obviously, the existence of a bicoloring of $G$ implies (d). We leave to the reader the easy proof of

Proposition 4.2. Every PEG has precisely two bicolorings obtained from each other by interchanging Black and White.

A bicolored planar Eulerian graph (BPEG) is a triple $G=(V, E, f)$ where $f$ is a bicoloring of the PEG $(V, E)$.

With a BPEG $G$ we associate three types $b_{G}, d_{G}, w_{G}$ defined as follows:

$$
\begin{aligned}
b_{G}(n) & =\mid\{U: U \text { is a black } G \text {-region and } c(U)=n\} \mid, \\
d_{G}(m) & =\mid\{v: v \text { is a } G \text {-vertex and } d(v)=2 m\} \mid, \\
w_{G}(l) & =\mid\{U: U \text { is a white } G \text {-region and } c(U)=l\} \mid .
\end{aligned}
$$

The following theorem provides the connection between PEGs and the present context. 
TheOREM 3. Let $G$ be a BPEG. Then $P\left(b_{G}, d_{G}, w_{G}\right)$.

The following illustrated examples deomonstrate a few applications of Theorem 3. EXAMPLE 1. The bicolored planar Eulerian graphs listed in Figure 1 constitute, by Theorem 3, proofs of Propositions 3.5-3.7.

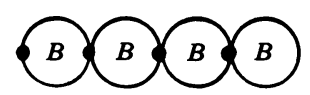

(a1)

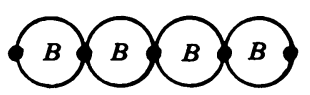

(a2)

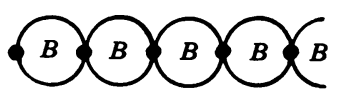

(a3)

By (a1), $P\left(1^{*}+m \cdot 2^{*}, 1^{*}+m \cdot 2^{*},(2 m+1)^{*}\right)(m=3)$.

By (a2), $P\left(m \cdot 2^{*}, 2 \cdot 1^{*}+(m-1) \cdot 2^{*},(2 m)^{*}\right)(m=4)$.

By (a3), $P\left(\boldsymbol{\aleph}_{0} \cdot 2^{*}, 1^{*}+\boldsymbol{\aleph}_{0} \cdot 2^{*}, \boldsymbol{\aleph}_{0}^{*}\right)$.

Proposition 3.5 follows.

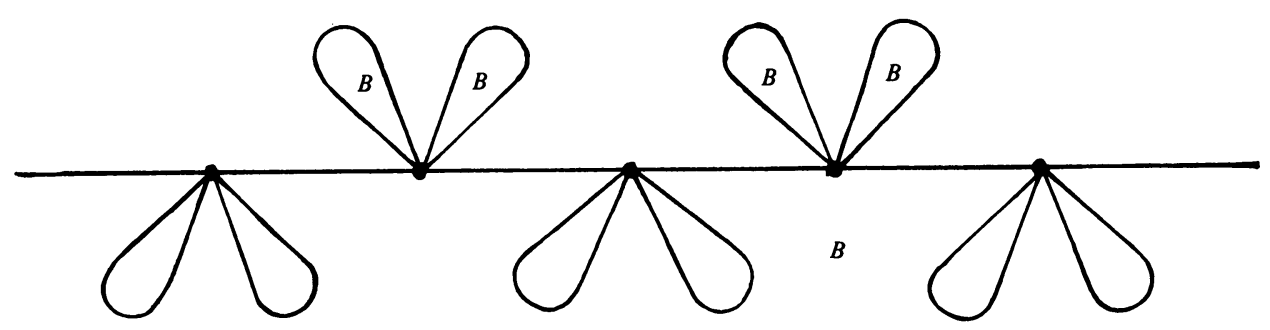

(b)

$P\left(\boldsymbol{\aleph}_{0} \cdot 1^{*}+\boldsymbol{\aleph}_{0}^{*}, \boldsymbol{\aleph}_{0} \cdot m^{*}, \boldsymbol{\aleph}_{0} \cdot 1^{*}+\boldsymbol{\aleph}_{0}^{*}\right)(m=3)$.

Proposition 3.6 follows.

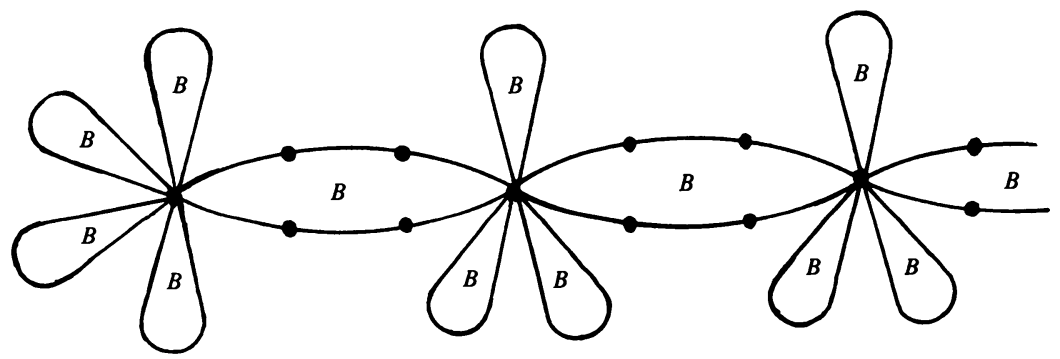

(c)

$P\left(\boldsymbol{\aleph}_{0} \cdot\left(1^{*}+n^{*}\right), \boldsymbol{\aleph}_{0} \cdot\left(1^{*}+m^{*}\right), \boldsymbol{\aleph}_{0}^{*}\right)(n=6, m=5)$.

Proposition 3.7 follows.

FIGURE 1

Example 2. By Figure 2, $P\left(\boldsymbol{\kappa}_{0} \cdot 3^{*}, 1^{*}+\boldsymbol{\aleph}_{0} \cdot 3^{*}, \boldsymbol{\aleph}_{0} \cdot 3^{*}\right)$ holds. Hence, by homogeneity, $P\left(\boldsymbol{\aleph}_{0} \cdot 3^{*}, \boldsymbol{\kappa}_{0} \cdot 1^{*}+\boldsymbol{\kappa}_{0} \cdot 3^{*}, \boldsymbol{\kappa}_{0} \cdot 3^{*}\right)$ and, by additivity, $P\left(\boldsymbol{\kappa}_{0} \cdot 3^{*}, \boldsymbol{\aleph}_{0} \cdot\left(1^{*}+3^{*}\right), \boldsymbol{\aleph}_{0} \cdot\left(1^{*}+3^{*}\right)\right)$; i.e., $Q(3,3)$, holds a special case of Proposition 3.8.

\footnotetext{
${ }^{5}$ In fact, $Q(n, n)$ is a trivial relation for all $n \in N^{+}$; but this derivation of $Q(3,3)$ models the derivation of $Q(n, m)$ for arbitrary $n, m$ specified in Propositions 3.8 and 3.9.
} 


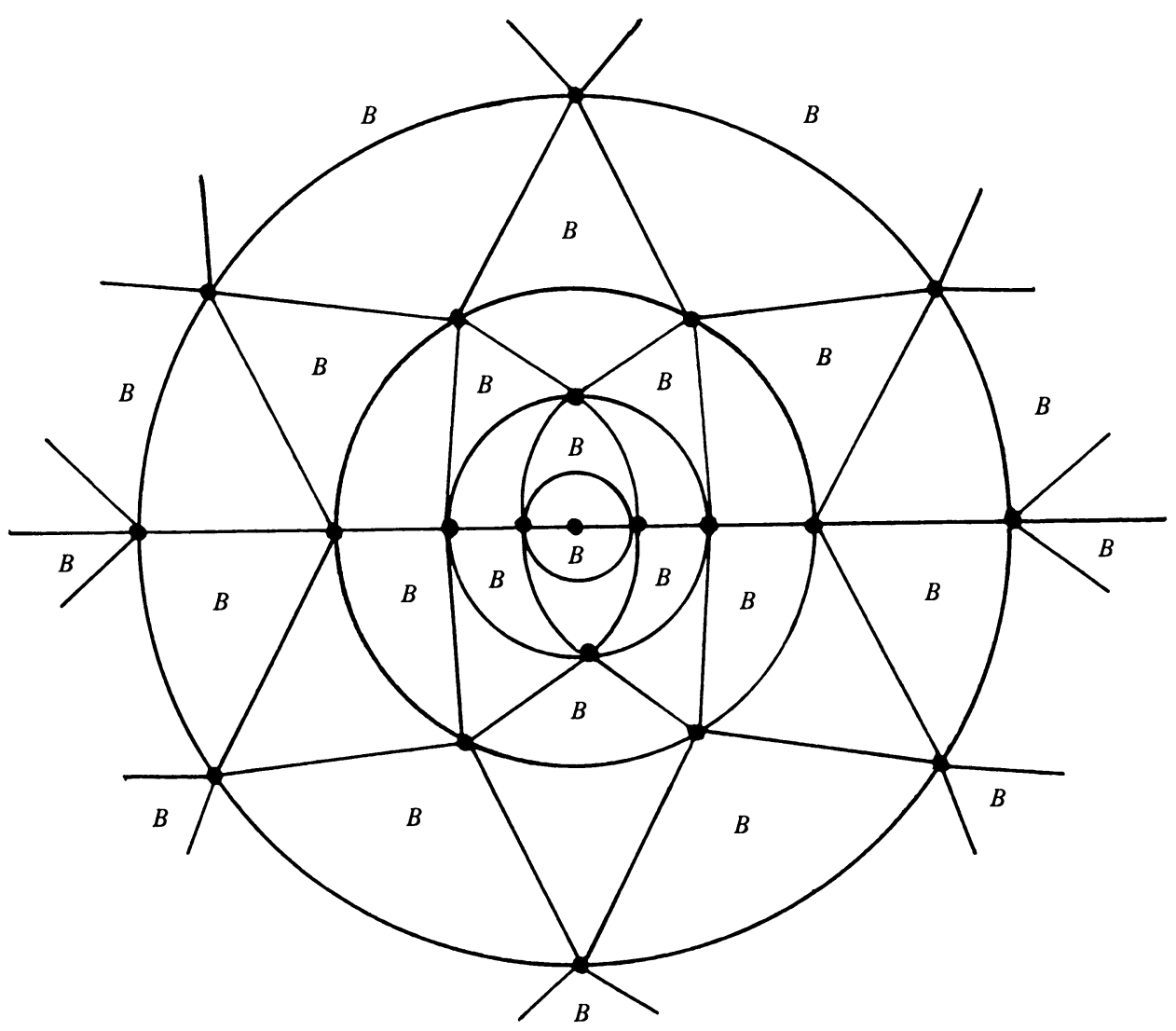

FIGURE 2

EXAmple 3. By Figure $3, P\left(\boldsymbol{\aleph}_{0} \cdot 4^{*}, \boldsymbol{\aleph}_{0} \cdot 2^{*}, 1^{*}+\boldsymbol{\aleph}_{0} \cdot 4^{*}\right.$ ) holds. Hence (see Example 2), $Q(2,4)$ holds, a special case of Proposition 3.9.

Proof of Theorem 3. Let $G$ be a bicolored PEG. We shall derive from $G$ a directed graph $D$ whose edges are colored $\eta, \zeta$ such that:

1. Every vertex $v$ of $D$ is the tail of just one $\eta$-edge and just one $\zeta$-edge, and it is the head of just one $\eta$-edge and just one $\zeta$-edge.

By $1, D$ defines two permutations $\eta$ and $\zeta$ of the set of its vertices. Let $\xi=\eta \zeta$.

2. $\bar{\xi}=b_{G}, \bar{\eta}=d_{G}, \bar{\zeta}=w_{G}$.

Thus, the existence of $D$ establishes $P\left(b_{G}, d_{G}, w_{G}\right)$.

First step. Transform $G$ into another bicolored PEG $\tilde{G}$, as follows:

(1) Around each vertex $v \in V$ draw a small circle $C_{v}$ so that the circles are disjoint.

(2) Choose $\frac{1}{2} d(v)$ new points on $C_{v}$-the new v-points-one in each white region that $C_{v}$ meets. $V_{\tilde{G}}$ is the set of all new $v$-points for every $v \in V_{G}$.

(3) Let $e \in E_{G}$, let $U$ be the white region touching $e$, and let $v, v^{\prime} \in V_{G}$ be the (not necessarily distinct) endpoints of $e$. Let $u$ and $u^{\prime}$ be the new $v$-point on $C_{v} \cap U$ and 


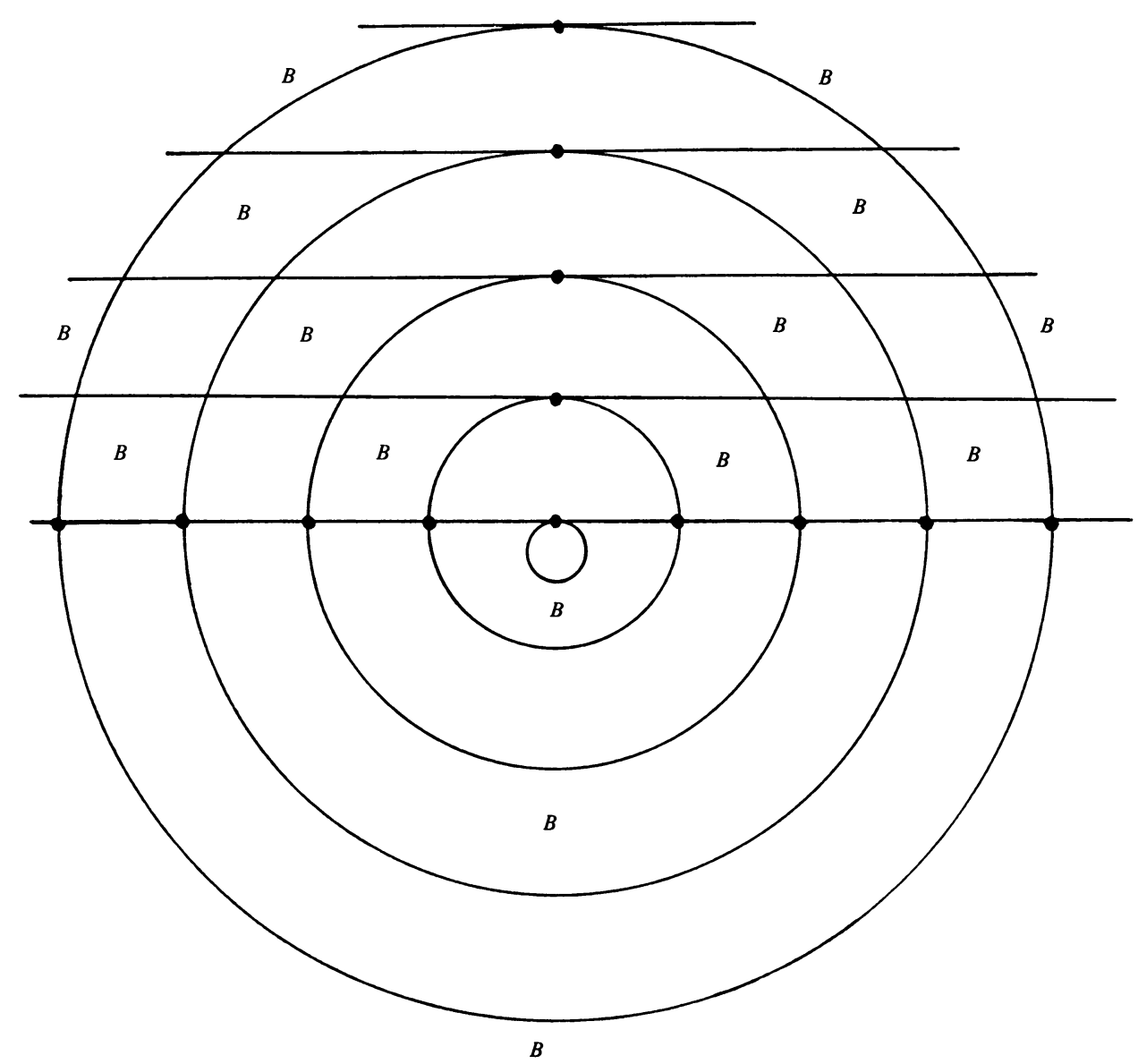

FIGURE 3

the new $v^{\prime}$-point on $C_{v^{\prime}} \cap U$. (Notice that $u=u^{\prime}$ if and only if $v=v^{\prime}$ and $e$ encloses a white $G$-region.) Connect $u$ and $u^{\prime}$ by a curve $e^{\prime}$ lying in $U$, intersecting $C_{v}$ in $u$ and $C_{v^{\prime}}$ in $u^{\prime} . E_{\tilde{G}}$ consists of all edges $e^{\prime}$ so obtained, together with all $C_{v}$-arcs determined by the new $v$-points for each $v \in V$.

(4) Color white the $\tilde{G}$-regions included in white regions of $G$ and the interiors of the circles $C_{v}$. Color black the other $\tilde{G}$-regions.

Second step. Color the $\tilde{G}$-edges by $\eta$ and $\zeta$ as follows: Color the $C_{v}$-arcs in $E_{\tilde{G}}(v \in V)$ by $\eta$, and all other $\tilde{G}$-edges by $\zeta$.

Third step. Obtain $D$ from $\tilde{G}$ by orienting its edges as follows: Let $\tilde{e}$ be any $\tilde{G}$-edge. Then $\tilde{e}$ is so oriented, that the white region is on the right (so that the circumferences of the bounded white regions are oriented clockwise). By Proposition 2.0 this definition makes sense.

We leave it to the reader to check that 1 and 2 hold. 

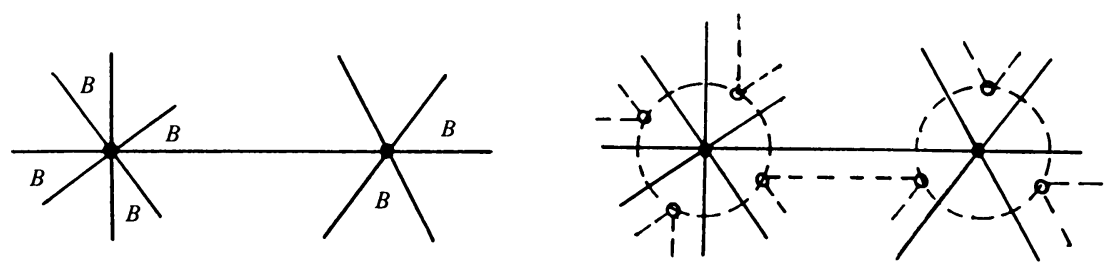

(0) Configuration in $G \quad$ (1) Introducing the circles $C_{v}$ and the new $v$-points,

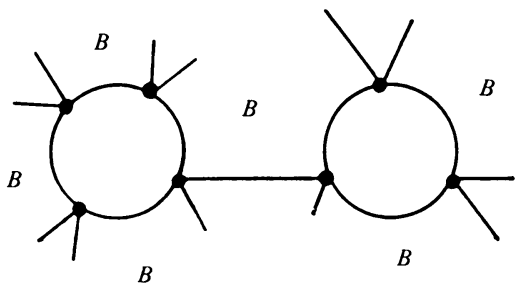

(2) The arising configuration in $\tilde{G}$

Figure 4

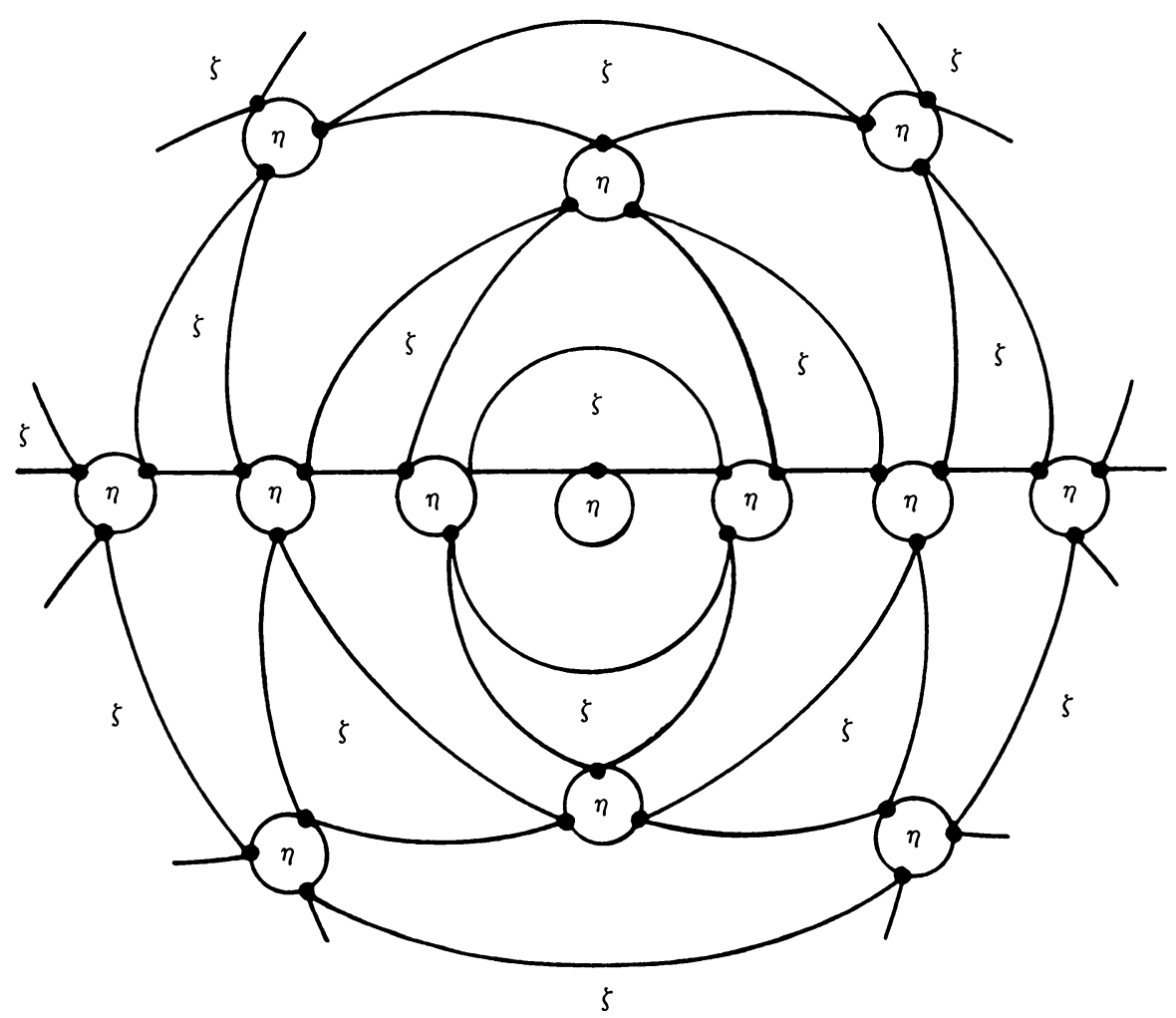

The digraph $D$ derived by the proof of Theorem 3 from the BPEG $G$ in Figure 2 is given in Figure 5 . The edges are so colored and oriented that an edge touching a region marked $\zeta(\eta)$ is marked $\zeta(\eta)$ and the circuits bounding the marked regions are oriented clockwise.

FigURE 5 
5. The planar graphs establishing Theorem 1. In this section we use Theorem 3 to prove Propositions 3.8 and 3.9, thereby completing the proof of Theorem 1 . These propositions follow by Lemma 1 from

Proposition 5.0. 1. $P\left(\boldsymbol{\aleph}_{0} \cdot n^{*}, 1^{*}+\boldsymbol{\aleph}_{0} \cdot m^{*}, \boldsymbol{\aleph}_{0} \cdot l^{*}\right)$ holds for all $n, m, l \in N$, $2<n, m, l$.

2. $P\left(\boldsymbol{\aleph}_{0} \cdot m^{*}, \boldsymbol{\aleph}_{0} \cdot 2^{*}, 1^{*}+\boldsymbol{\aleph}_{0} \cdot l^{*}\right)$ holds for all $m, l \in N, 3<m, l$.

We shall establish this proposition by showing that suitable bicolored planar Eulerian graphs (BPEGs) exist. At this point, the reader may wish to consult again Figures 2 and 3, which establish assertions 1 and 2 with $n=m=l=3$ and $m=l=4$, respectively.

Our method of proof is also indicated by the above figures. We obtain the required BPEGs as an increasing union of finite bicolored disc-maps, taking care in transitions from one such map to the next that the new black regions, new degrees defined for internal vertices, and new white regions are as required by the triple of types $b_{G}, d_{G}, w_{G}$ in question. A somewhat more general proposition will be proved, namely,

Proposition 5.1. Let $\left(n_{i}\right)_{i \in N},\left(m_{j}\right)_{j \in N},\left(l_{k}\right)_{k \in N}$ be sequences of integers satisfying at least one of the following two conditions.

1. $m_{1}=1 ; 2<n_{i}, m_{j}, l_{k}, i, j, k \in N, 1<j$,

2. $l_{1}=1 ; 3<n_{i}, l_{k}, 1<m_{j}, \quad i, j, k \in N, 1<k$.

Then there is a bicolored planar Eulerian graph $G$ and enumerations $\left(B_{i}\right)_{i \in N}$, $\left(v_{j}\right)_{j \in N},\left(W_{k}\right)_{k \in N}$ of the black G-regions, the set of $G$-vertices, and the white G-regions, respectively, so that $c\left(B_{i}\right)=n_{i}, d\left(v_{j}\right)=2 m_{j}, c\left(W_{k}\right)=l_{k} ; i, j, k \in N$.

(Recall that $c\left(B_{i}\right), d\left(v_{j}\right), c\left(W_{k}\right)$ are the number of edges of the circuit enclosing $B_{i}$, the degree of $v_{j}$ and the number of the edges of the circuit enclosing $W_{k}$, respectively.)

The proof of Proposition 5.1 requires some more notation. For brevity, take the plane to be the set of complex numbers. For a positive integer $n$, let $C_{n}=\{z$ : $|z|=n\}, D_{n}=\{z:|z| \leqslant n\}$. By an $n$-disc graph ( $n$-DG) we shall mean a triple $G=\left(V_{G}, E_{G}, f_{G}\right)$, satisfying the following conditions.

(A) $\left(V_{G}, E_{G}\right)$ is a planar graph such that

(A0) $G_{*}=V_{G} \cup \bigcup_{e \in E_{G}} e$ is connected;

(A1) $C_{n} \subseteq G_{*} \subseteq D_{n}$;

(A2) for $v \in V_{G} \cap C_{n}, d(v) \leqslant 4$.

(B) $f_{G}$ is a B-W coloring of the bounded $G$-regions; that is, every edge $e \in E_{G}$ not on $C_{n}$ touches a black and a white region.

Notice that by (a), (b) and (A1), $V_{G}$ and $E_{G}$ are finite, and $d_{G}(v) \geqslant 2$ for $v \in V_{G} \cap C_{n}$; and by (A0) and (B), $d_{G}(v)>0$ is even for every $v \in V_{G} \backslash C_{n}$ (such a vertex $v$ will be called an inner vertex of $G$ ).

We say that the $(n+1)$-DG $G^{\prime}=\left(V^{\prime}, E^{\prime}, f^{\prime}\right)$ extends the $n$-DG $G=(V, E, f)$ if $V=V^{\prime} \cap D_{n}, E=\left\{e \in E^{\prime}: e \subseteq D_{n}\right\}$ and $f^{\prime}(U)=f(U)$ for every bounded $G$-region $U$. 
Our proof of Proposition 5.1 consists in showing that a bicolored planar graph $G$ with the required properties can be constructed as the union $G=\bigcup_{n=1}^{\infty} G_{n}$, where for each $n, G_{n}=\left(V_{n}, E_{n}, f_{n}\right)$ is an $n$-DG and $G_{n+1}$ extends $G_{n} . f_{n}$ will be specified by a pair of sequences $\left(B_{i}\right)_{i}$ and $\left(W_{k}\right)_{k}$ enumerating the black and white $G_{n}$-regions.

We shall refer to the assumption that the condition 1 (2) of Proposition 5.1 holds as "Case 1" ("Case 2").

1. Base of the inductive construction. Let $G_{1}=\left(V_{1}, E_{1}, f_{1}\right)$ be the 1-DG shown in Figure 6. Formally, $V_{1}=\{-1,0,1\} \cup V_{1}^{+} \cup V_{1}^{-}$, where $V_{1}^{+}\left(V_{1}^{-}\right)$is the set of vertices on the upper (lower) half of the circle $C_{1}$.

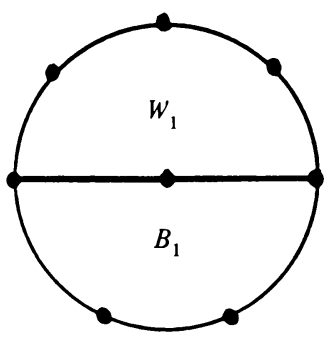

Case 1. With $n_{1}=5, l_{1}=6$.

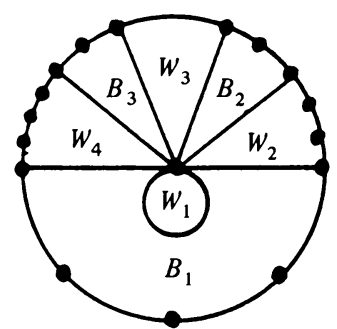

Case 2. With $\left(n_{1}, n_{2}, n_{3}\right)=(7,4,4)$,

$$
m_{1}=4,\left(l_{2}, l_{3}, l_{4}\right)=(5,3,6) \text {. }
$$

FIGURE 6

Case 1. $V_{1}^{+}\left(V_{1}^{-}\right)$is chosen so that $\left|V_{1}^{+}\right|=l_{1}-3\left(\left|V_{1}^{-}\right|=n_{1}-3\right) . E_{1}$ consists of the closed arcs of $C_{1}$ determined by $V_{1} \cap C_{1}$, and the radial segments connecting 0 with $1,-1$, i.e. the closed intervals $[0,1],[-1,0]$.

Case 2. Step 1 . We specify a subset $E^{\prime}$ of $E_{1}$. Let $E^{\prime}$ consist of $[-1,0],[0,1]$ and a loop $e$ with endpoint 0 , all of whose nonzero points lie in the interior of the lower half of $D_{1}$.

Step 2. We specify a subset $V^{\prime}$ of $V^{+}$. Choose arbitrarily $2\left(m_{1}-2\right)$ points in $C_{1} \cap\{z: \operatorname{Im}(z)>0\}$.

Step 3. We specify another subset $E^{\prime \prime}$ of $E_{1}$. Let $E^{\prime \prime}$ consist of all radial segments connecting 0 with vertices in $V^{\prime}$.

Step 4. We assign colors to the regions into which $D_{1}$ was divided. Let $W_{1}$ be the region bounded inside the loop $e$, let $B_{1}$ be the other region in the lower half of $D_{1}$, and let $W_{2}, B_{2}, \ldots, W_{m_{1}-1}, B_{m_{1}-1}, W_{m_{1}}$ be enumeration of the triangular regions into which the upper half of $D_{1}$ was divided in Step 3, in cyclical (say, positive) order.

Step 5. We specify another subset $V^{\prime \prime}$ of $V_{1}^{+} \cup V_{1}^{-}$by choosing arbitrarily extra points on $C_{1}$ so as to satisfy the equalities $C\left(W_{1}\right)=l_{1}, \ldots, C\left(W_{m_{1}}\right)=l_{m_{1}}, C\left(B_{1}\right)=$ $n_{1}, \ldots, C\left(B_{m_{1}-1}\right)=n_{m_{1}-1}$. Let $E^{\prime \prime \prime}$ be the set of arcs into which $C_{1}$ is divided by vertices chosen on $C_{1}$.

Step 6. Let $V_{1}=\{-1,0,1\} \cup V^{\prime} \cup V^{\prime \prime} ; E_{1}=E^{\prime} \cup E^{\prime \prime} \cup E^{\prime \prime \prime}$, and $f_{1}$ as determined in Step 4.

2. Induction step. Assume that $n$-DG $G_{n}=\left(V_{n}, E_{n}, f_{n}\right)$ is defined. Let $B_{1}, \ldots, B_{p}$; $v_{1}, \ldots, v_{q} ; W_{1}, \ldots, W_{r}$ enumerate the black $G_{n}$-regions, the inner $G_{n}$-vertices and the 
white $G_{n}$-regions. Let $u_{1}=v_{q+1}, \ldots, u_{s}=v_{q+s}$ enumerate $V_{n} \cap C_{n}$ in positive cyclic order, let $\left\lfloor u_{i}, u_{i+1}\right\rfloor \in E_{n}$ denote the arc on $C_{n}$ from $u_{i}$ to $u_{i+1}\left(u_{s+1}=u_{1}\right)$ and let $X_{i} \in\{B, W\}$ be the color of the bounded $G$-region touching $\left\lfloor u_{i}, u_{i+1}\right\rfloor$. We now extend $G_{n}$ to $G_{n+1}$ as follows.

Case 1. Step 1. For $t=1, \ldots, s$ let $y_{t}$ denote the intersection of the ray from 0 through $u_{t}$ by $C_{n+1}$. Choose $y_{t}^{+} \in C_{n+1}$ between $y_{t}$ and $y_{t+1}$ (where $y_{s+1}=y_{1}$ ) $\left(y_{t}^{+}=y_{t+1}\right.$ is possible, if so required in Step 3). Let $y_{t}, y_{t}^{+} \in V_{n+1}$. Let $\left[u_{t}, y_{t}\right],\left[u_{t}\right.$, $\left.y_{t}^{+}\right]$belong to $E_{n+1}$ (where $[x, y]$ denotes the straight line segment from $x$ to $y$ ).

Step 2. Color the regions bounded by the segments $\left[u_{t}, y_{t}^{+}\right],\left[u_{t+1}, y_{t+1}\right]$ and the arcs $\left\lfloor u_{t}, u_{t+1}\right\rfloor,\left\lfloor y_{t}^{+}, y_{t+1}\right\rfloor$ properly; i.e., $B$ if $X_{t}=W$ and $W$ otherwise. Let $B_{p+1}, \ldots, B_{p^{\prime}} ; W_{r+1}, \ldots, W_{r^{\prime}}$ denote the black and white new regions so obtained.

Step 3. Let $i \in\left\{p+1, \ldots, p^{\prime}\right\}\left(k \in\left\{r+1, \ldots, r^{\prime}\right\}\right)$. Let $B_{i}\left(W_{k}\right)$ touch the arc $\left\lfloor u_{t}, u_{t+1}\right\rfloor \subseteq C_{n}$. Let $y_{t}^{+}=y_{t+1}$ if $n_{i}=3\left(l_{k}=3\right)$. Otherwise, let $\left\lfloor y_{t}^{+}, y_{t+1}\right\rfloor$ be a nontrivial arc on $C_{n+1}$ containing $n_{i}-4\left(l_{k}-4\right)$ new vertices. (This ensures $c\left(B_{i}\right)=n_{i}, c\left(W_{k}\right)=l_{k}, i=p+1, \ldots, p^{\prime} ; k=r+1, \ldots, r^{\prime}$.)

Step 4. Let $4 \leqslant m_{t}^{\prime} \leqslant 6$ be the degree of $u_{t}$ in the graph drawn so far, $t=1, \ldots, s$. Choose $2 m_{q+t}-m_{t}^{\prime}$ points on the arc $\left\lfloor y_{t}, y_{t}^{+}\right\rfloor$. These points are vertices in $V_{n+1} \cap C_{n+1}$. Every such point is then connected to $u_{t}$ by a straight line segment. All these segments belong to $E_{n+1}$. (This ensures $d\left(v_{j}\right)=2 m_{j}, j=q+1, \ldots, q+s$.) In this step the triangular regions with vertices $u_{t}, y_{t}, y_{t}^{+}$are subdivided into triangular regions with common vertex $u_{t}$ and the two other vertices on $C_{n+1}$.

Step 5. Color properly the triangular regions obtained in Step 4 Black and White. Denote by $B_{p^{\prime}+1}, \ldots, B_{p^{\prime \prime}}, W_{r^{\prime}+1}, \ldots, W_{r^{\prime \prime}}$ the new triangular colored regions.

Step 6. Add extra vertices in $V_{n+1} \cap C_{n+1}$ as needed to ensure $c\left(B_{i}\right)=n_{i}$, $c\left(W_{k}\right)=l_{k}$ for $i=p^{\prime}+1, \ldots, p^{\prime \prime}, k=r^{\prime}+1, \ldots, r^{\prime \prime}$. Let $E_{n+1}$ contain all arcs determined on $C_{n+1}$ by $V_{n+1} \cap C_{n+1}$.

$G_{n+1}$ is now defined.

Case 2. Here our induction hypothesis on $u_{1}, \ldots, u_{s}$ includes the extra assumption:

$$
\text { if } t \neq t^{\prime} \text { and } d\left(u_{t}\right)=d\left(u_{t^{\prime}}\right)=4 \text {, then }\left|t-t^{\prime}\right|>1 \text {. }
$$

Step 0 . Declare the degrees of $u_{1}, \ldots, u_{s}$ to be $2 m_{q+1}, \ldots, 2 m_{q+s}$, respectively.

Proceed through Steps 1-6 of Case 1, modified as required by Step 0. In particular, in Step 1, no $y_{t}, y_{t}^{+} \in C_{n+1}$, are defined if $m_{q+t}=2$ and the degree of $u_{t}$ is 4. ((*) and $n_{t}, l_{t}>3$ are used in Step 3. In Step 6, $(*)$ is carried over to $G_{n+1}$ by $n_{t}, l_{t}>3$.)

It is easily checked that the $(n+1)$-DG $G_{n+1}=\left(V_{n+1}, E_{n+1}, f_{n+1}\right)$ so defined extends $\left(G_{n}, f_{n}\right)$, and that the union graph is as required by Proposition 5.1.

The proof of Theorem $1^{\prime}$ is complete.

6. The covering number of $H_{0}$. In this section we briefly indicate the proof that $\operatorname{cn}\left(H_{0}\right)=2$ if $\nu>0$. In [ACM] an analogous but stronger result was obtained for $H_{\tau}$ with $0<\tau<\nu$. For a group $G$ let the extended covering number of $G, \operatorname{ecn}(G)$, [AHS] denote the smallest integer $k+1$ such that whenever $C_{1}, \ldots, C_{k+1}$ are nonunit classes of $G$, we have $G=C_{1} \cdots C_{k+1}$. It is not hard to show that $k+1 \geqslant \operatorname{ecn}(G)$ iff, whenever $C_{1}, \ldots, C_{k}$ are nonunit classes of $G$, we have $C_{1} \cdots C_{k} \supseteq G \backslash 1$, where 
1 denotes the unit-class. Thus, if $k+1=\operatorname{ecn}(G)$ and $\mathbf{1} \subseteq C^{k}$ for every class $C \neq \mathbf{1}$ then $\operatorname{cn}(G) \leqslant k$.

In $[\mathbf{A C M}]$ it is actually shown that if $\nu>0,0<\tau<\nu$, then $\operatorname{ecn}\left(H_{\tau}\right)=3$ (whence $\operatorname{cn}\left(H_{\tau}\right)=2$ follows). This result is true also when $\tau=0$. We state it as

THEOREM 4. Let $\nu>0$. Then $\operatorname{ecn}\left(H_{0}\right)=3$.

Corollary. $\operatorname{cn}\left(H_{0}\right)=2$.

For types $t, s$ let $t \equiv s$ iff $t=t^{\prime}+r, s=s^{\prime}+r$ for some $t^{\prime}, s^{\prime}, r \in T$, with $\left|t^{\prime}\right|=\left|s^{\prime}\right|<\boldsymbol{N}_{0}$.

Proposition 6.0 [M, Theorem 4]. Let $\nu \geqslant 0$, let $G=S / S^{0}$ and let $\xi, \eta \in S$. Then $\left[\xi S^{0}\right]_{G}=\left[\eta S^{0}\right]_{G}$ iff $\bar{\xi} \equiv \bar{\eta}$.

Define two sets of countable types $U_{0}, U_{1}$ by $t \in U_{0} \Leftrightarrow t=\boldsymbol{\aleph}_{0} \cdot 1^{*}+t^{\prime}$, where $t^{\prime}(1)=0$ and $n>1 \Rightarrow t(n) \in\left\{0, \boldsymbol{\aleph}_{0}\right\}, s \in U_{1} \Leftrightarrow s=\boldsymbol{\aleph}_{0} \cdot 1^{*}+s^{\prime}$, where $s^{\prime}(1)=$ $s^{\prime}(2)=s^{\prime}(3)=0$, and $n>1 \Rightarrow s^{\prime}(n)<\boldsymbol{\aleph}_{0}$, and $\Sigma_{n>3} n s(n) \in\left\{0, \boldsymbol{\aleph}_{0}\right\}$. Let $U=U_{0}$ $+U_{1}$. By a $U$-type $\left(U_{i}\right.$-type) we shall mean a type lying in $U\left(U_{i}, i=0,1\right)$. From Proposition 6.0 follows

Proposition 6.1. Let $C$ be a class in $H_{0}$. Then $C=\left[\xi S^{0}\right]$ where $\xi \in S$ satisfies $\bar{\xi}=\aleph_{v} \cdot 1^{*}+r$, r a U-type.

Theorem 4 follows by Lemma 1 from

Proposition 6.2. Let $r, s, t$ be nonunit $U$-types. Then $P(r, s, t)$.

Define sets $V_{i}^{\prime}$ of countable types, $i=1,2,3$ by $v^{\prime} \in V_{1}^{\prime}$ iff $v^{\prime}=\boldsymbol{\aleph}_{0} \cdot n^{*}, 1<n$ $<\boldsymbol{\aleph}_{0} ; v^{\prime} \in V_{2}^{\prime}$ iff $v^{\prime}=m \cdot \boldsymbol{\aleph}_{0}^{*}, m \in N ; v^{\prime} \in V_{3}^{\prime}$ iff $v^{\prime}=\sum_{n \in A} n^{*}$, where $A \subseteq$ $N \backslash\{1,2,3\},|A|=\boldsymbol{\aleph}_{0}$. Let $V^{\prime}=V_{1}^{\prime} \cup V_{2}^{\prime} \cup V_{3}^{\prime}$ and define $V_{i}, V$ by $v \in V_{i}$ iff $v=\boldsymbol{\aleph}_{0} \cdot 1^{*}+v^{\prime}, v^{\prime} \in V_{i}^{\prime}, i=1,2,3 ; v \in V$ iff $v=\boldsymbol{\aleph}_{0} \cdot 1^{*}+v^{\prime}, v^{\prime} \in V^{\prime}$.

Obviously, $V \subseteq U$ and every $u \in U$ is a sum of members of $V$. Moreover, if $u \notin V_{2}$, i.e., $u$ is not $\boldsymbol{\aleph}_{0} \cdot 1^{*}+m \cdot \boldsymbol{\aleph}_{0}^{*}$ for some $1 \leqslant m \leqslant \boldsymbol{\aleph}_{0}$, then $u$ is a sum of countably many members of $V$. To establish Proposition 6.2 we shall need the following recent result of Droste.

Proposition 6.3 [D, Theorem A]. $P(r, s, t)$ holds whenever $|r|=|s|=|t|=$ $\sum_{n>1} n t(n)=\boldsymbol{\aleph}_{0}$ and $r\left(\boldsymbol{\aleph}_{0}\right), s\left(\boldsymbol{\aleph}_{0}\right)>0$.

Proposition 6.4. $P\left(v_{1}, v_{2}, v_{3}\right)$ holds for every $v_{1}, v_{2}, v_{3} \in V$.

Proof. We leave it to the reader to fill in the details of the proofs of the following inclusions:

$$
\begin{array}{ll}
V_{1} \times V_{1} \times V_{1} \subseteq P & (\text { by Lemma } 3), \\
V_{1} \times V_{1} \times V_{2} \subseteq P & (\text { modify proof of Propostion 3.7 (Figure 1)) } \\
V_{1} \times V_{1} \times V_{3} \subseteq P & (\text { use 5.1, 3.5 and Figure 7) }
\end{array}
$$


$V_{1} \times V_{2} \times V_{2} \subseteq P \quad$ (modify proof of Proposition 3.6 (Figure 1)) $V_{1} \times V_{2} \times V_{3} \subseteq P \quad$ (modify proof of Proposition 3.6 (Figure 1)), $V_{1} \times V_{3} \times V_{3} \subseteq P \quad$ (use Proposition 5.1), $V_{2} \times V_{2} \times V_{2} \subseteq P \quad$ (use Proposition 6.3), $V_{2} \times V_{2} \times V_{3} \subseteq P \quad$ (modify proof of Proposition 3.6 (Figure 1)), $V_{2} \times V_{3} \times V_{3} \subseteq P \quad$ (modify proof of Proposition 3.7 (Figure 1)), $V_{3} \times V_{3} \times V_{3} \subseteq P \quad$ (use Proposition 5.1).

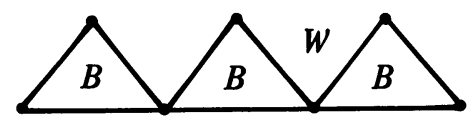

(0) $P\left(k \cdot 3^{*},(k+2) \cdot 1^{*}+(k-1) \cdot 2^{*},(3 k)^{*}\right)$

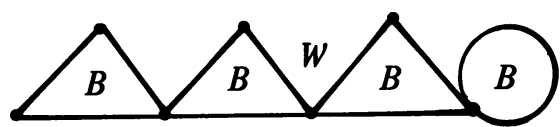

(1) $P\left(1^{*}+k \cdot 3^{*} ;(k+1) \cdot 1^{*}+k \cdot 2^{*},(3 k+1)^{*}\right)$

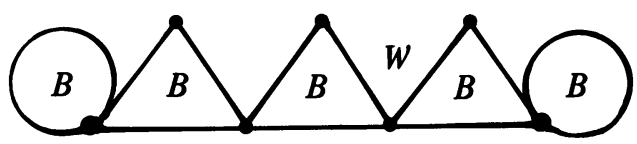

(2) $P\left(2^{*}+k \cdot 3^{*}, k \cdot 1^{*}+(k+1) \cdot 2^{*},(3 k+2)^{*}\right)$

The relations (0), (1), (2) are established by the indicated bicolored Eulerian graphs (for $k=3$ ). (0), (1), (2) imply $P\left(\aleph_{0} \cdot\left(1^{*}+3^{*}\right), \aleph_{0} \cdot\left(1^{*}+2^{*}\right), t\right)$ for any type $t$ with $t(2)=t\left(\aleph_{0}\right)=0,|t|=t(1)=$ $\Sigma_{n>1} t(n)=\kappa_{0}$.

\section{Figure 7}

Proof of Proposition 6.2. Let $r, s, t \in U$. We distinguish three cases.

Case 1. $r, s, t \notin V_{2}$. Then we can put $r=\sum_{n \in N} r_{n}, s=\sum_{n \in N} s_{n}, t=\sum_{n \in N} t_{n}$ with $r_{n}, s_{n}, t_{n} \in V$ for all $n \in N$. $P(r, s, t)$ follows by Proposition 6.4 and Lemma 1 .

Case 2. $r \in V_{2}, s, t \notin V_{2}$.

2.1. $r\left(\boldsymbol{\aleph}_{0}\right)=s\left(\boldsymbol{\aleph}_{0}\right)=0$ : modify the proof of Proposition 3.7.

2.2. $s\left(\boldsymbol{\aleph}_{0}\right)>0$ : use 6.3.

Case 3. $r, s \in V_{2}$ : use 6.3.

The proof of Theorem 4 is complete.

We conclude by summarizing the situation when $\nu=0$.

THEOREM 5. Let $\nu=0$. Then $\operatorname{ecn}\left(H_{0}\right)=4$. 
Proof. ecn $\left(H_{0}\right) \geqslant 4$ since $P\left(\boldsymbol{\aleph}_{0} \cdot 2^{*}, \boldsymbol{\aleph}_{0} \cdot 2^{*}, \sum_{n \in N} n^{*}\right)$ does not hold (see [ACM]). ecn $\left(H_{0}\right) \leqslant 4$ follows from a recent result of M. Droste, [D, Corollary 4.10] which asserts that $[x] \subseteq[\xi] \cdot[\eta] \cdot[\zeta]$ whenever $x, \xi, \eta, \zeta$ are permutations of a countable set, each moving infinitely many elements.

Proposition 6.5. Let $\nu=0$ and let $C$ be a class in $H_{0}$. Then $C \subseteq C^{2}$, and so by $C=C^{-1}, \mathbf{1} \subseteq C^{3}$.

Proof. Let $C \neq 1$ be a class in $H_{0}$. Then by Proposition $6.0, C=\xi S^{0}$, where $\bar{\xi} \in U$ or else $\bar{\xi}(1)<\boldsymbol{\aleph}_{0}$ and $\sum_{1<n} n \bar{\xi}(n)=\boldsymbol{\aleph}_{0}$. In the first case $C^{2} \subseteq C$, as $P(\bar{\xi}, \bar{\xi}, \bar{\xi})$ holds by Proposition 6.2. In the second case we distinguish two cases.

Case 1. $\bar{\xi}\left(\boldsymbol{\aleph}_{0}\right)>0$. Then $P(\bar{\xi}, \bar{\xi}, \bar{\xi})$ holds by Proposition 6.3 , and so again $C^{2} \subseteq C$.

Case 2. $\bar{\xi}\left(\boldsymbol{\aleph}_{0}\right)=0$. We may assume $\bar{\xi}(2) \in\left\{0, \boldsymbol{\aleph}_{0}\right\}$ (if $0<\bar{\xi}(2)=m<\boldsymbol{\aleph}_{0}$ replace $\xi$ by $\xi^{\prime}$ with $\bar{\xi}^{\prime}(1)=\bar{\xi}(1)+2 m, \bar{\xi}^{\prime}(2)=0, \bar{\xi}^{\prime}(n)=\bar{\xi}(n)$ for $\left.n>2\right)$. Then $P(\bar{\xi}, \bar{\xi}, \bar{\xi})$ follows from $P\left(2 \cdot 2^{*}, 2 \cdot 2^{*}, 2 \cdot 2^{*}\right)$ and Proposition 5.1.

From Theorem 5 and Proposition 6.5 follows

THEOREM $6[\mathrm{ACM}]$. Let $\nu=0$. Then $\mathrm{cn}\left(H_{0}\right)=3$.

The proof of Theorem 6 outlined in [ACM] reduces it to the following proposition, which is of independent interest.

Proposition 6.6. Let $\xi$ be any permutation of a countable set moving infinitely many symbols. Then $[\xi] \subseteq[\xi]^{2}$.

This proposition follows from the argument for Proposition 6.5 and the relation $P(\bar{\xi}, \bar{\xi}, \bar{\xi})$, where $\bar{\xi}=2^{*}+(2 m)^{*}$, proved in [Hsu].

In view of Theorems $1,4,5$, a natural guess concerning the extended covering number of $H_{\nu}, \nu>0$, is that $\operatorname{ecn}\left(H_{\nu}\right)=3$. In fact, a general conjecture of J. Stavi supports this guess. It states

Conjecture (Stavi). Let $G$ be a finite simple group. Then $\operatorname{ecn}(G)=\operatorname{cn}(G)+1$.

Unexpectedly, it turns out that this guess is false (and so the word "finite" may not be omitted in Stavi's conjecture). We leave to the reader the proof that if $\nu>0$, $|B|=\aleph_{\nu}$, and if $\xi, \eta, \zeta \in S$ satisfy $\bar{\xi}=\boldsymbol{\aleph}_{\nu} \cdot 3^{*}, \bar{\eta}=\boldsymbol{\aleph}_{\nu} \cdot\left(1^{*}+2^{*}\right), \bar{\zeta}=\boldsymbol{\aleph}_{\nu} \cdot 2^{*}$ then $\left[\xi S^{\nu}\right] \cap\left(\left[\eta S^{\nu}\right] \cdot\left[\zeta S^{\nu}\right]\right)=\varnothing$. A full discussion of the relation $P$ on the classes of $H_{\nu}$ will appear elsewhere [M1].

ADDED IN PROOF. Recently S. Karni $[\mathbf{K}]$ found that $\operatorname{cn}\left(C_{3}\right)=3, \operatorname{ecn}\left(C_{3}\right)=5$ where $C_{3}$ is the sporadic finite group Conway 3 .

\section{REFERENCES}

[ACM] Z. Arad, D. Chilag and G. Moran, Groups with a small covering number, Products of Conjugacy Classes in Groups (Z. Arad and M. Herzog, Eds.), Lecture Notes in Math., Springer, Berlin and New York (to appear).

[AHSt] Z. Arad, M. Herzog and J. Stavi, Powers and products of conjugacy classes in groups, Products of Conjugacy Classes in Groups (Z. Arad and M. Herzog, Eds.), Lecture Notes in Math., Springer, Berlin and New York (to appear).

[D] M. Droste, Products of conjugacy classes of the infinite symmetric group, Discrete Math. 47 (1983), $35-48$. 
[F] R. H. Fox, On Fenchel's conjecture about F-groups, Mat. Tidsskr. B (1952), 61-65.

[G] A. B. Gray, Infinite symmetric and monomial groups, Ph. D. Thesis, New Mexico State University, Las Cruces, 1960.

[Hu] C. L. Hsu, The commutators of the alternating group, Sci. Sinica Ser. 14 (1965), 339-342.

[K] S. Karni, Covering numbers of small order and sporadic groups, Products of Conjugacy Classes in Groups (Z. Arad and M. Herzog, Eds.), Lecture Notes in Math., Springer, Berlin and New York (to appear).

[Mi] G. A. Miller, On the products of two substitutions, Amer. J. Math. 22 (1900), 185-190.

[M] G. Moran, Parity features for classes of the infinite symmetric group, J. Combin. Theory Ser. A 33 (1982), 82-98.

[M1] __ Products of conjugacy classes in some infinite simple groups (to appear).

[S] W. R. Scott, Group theory, Prentice-Hall, Englewood Cliffs, N. J., 1964.

Department of Mathematics, York University, Toronto, Canada

Current address: Department of Mathematics, University of Haifa, Haifa, Israel 